\title{
nature
}

physics

\section{A few holes to fill}

\author{
Once it seemed there were but a few holes in our understanding of physics. Today, we risk \\ crucial gaps opening up in the funding of physics research.
}

Few physicists have left their mark on science to quite the same extent as Max Planck: Planck's constant, the Planck scale and other Planck units, Planck's law for black-body radiation, the Max Planck Society and its 80 Max Planck Institutes across his native Germany.

Planck was born on 23 April 1858 this month marks his 150th anniversary. He arrived at the University of Munich in 1874 , to be told by his professor, Philipp von Jolly, that there was little point in pursuing an interest in physics. Jolly is credited with the words "In this field, almost everything is already discovered, and all that remains is to fill a few holes." Fortunately, Planck was undeterred. His motivation was not the potential for discovery, but a fascination with physics itself, a desire to understand all that was known about the very basis of nature.

In a pleasing twist to the story, by 1900 Planck's study of black-body radiation had led him to the notion of the quantization of energy. Now there were hardly a few holes to fill, but a gaping chasm of possibility. Planck had launched quantum theory.

Einstein's 1905 work on the photoelectric effect set the seal on the new physics, and in 1911 Planck and Walther Nernst organized the first of the conferences that became synonymous with the revolution in twentieth-century physics, sponsored by the Belgian industrialist Ernest Solvay. The rest, as they say, is history.

In 2008, we are in the early years of the much-touted 'century of biology'.

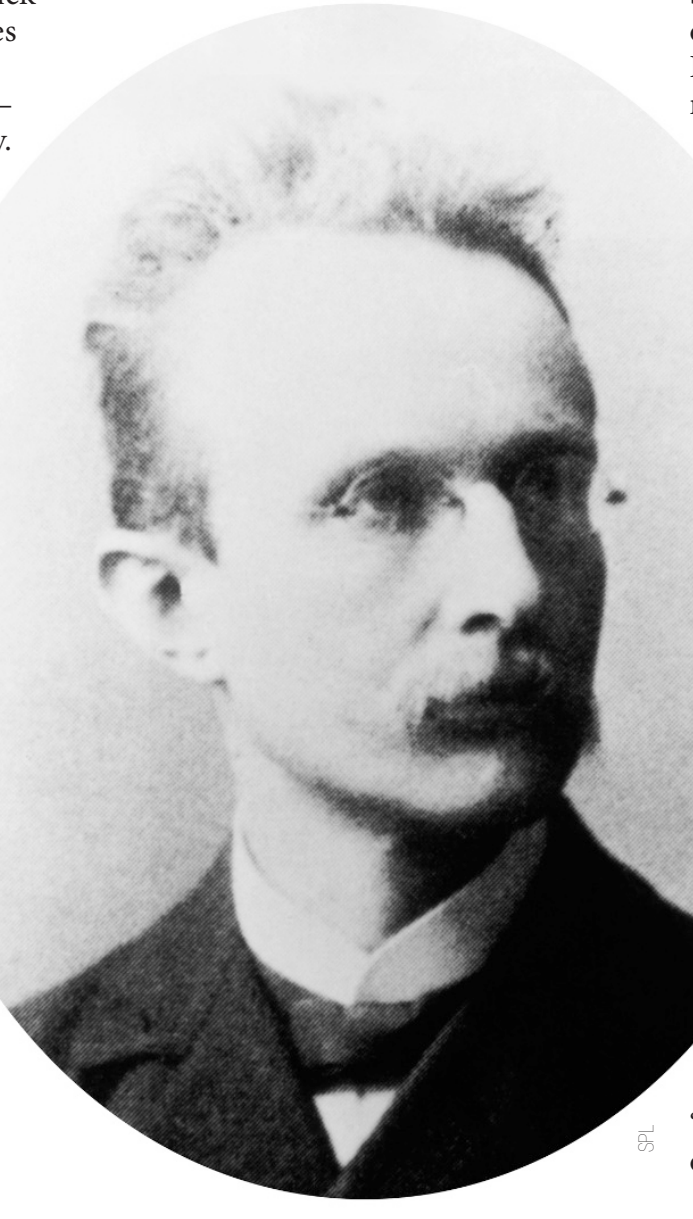

Physics, you could say, is history. Certainly, recent funding decisions, particularly in the USA and the UK, would suggest that physics does not enjoy a high priority in government circles. Once again, funding for US physics is trapped in a whirl of appropriations bills and budget shortfalls, the promises of the American Competitiveness Initiative unfulfilled. UK physicists are reeling from a $25 \%$ cut in new grants and wholesale pull-outs from projects such as the International Linear Collider; fundamental research in physics is carrying the can, it seems, for a misestimation of the operating costs of the Diamond synchrotron, a facility primarily of benefit to biology and materials science.

Jolly's words to Planck have new resonance. In an admittedly adverse economic climate, it would seem that many governments might be inclined to the view that there are only "a few holes" to fill in physics. But that would be to ignore the huge relevance of the subject to economic growth and development and, not least, Planck's example that small wrinkles in the fabric of understanding may prove to be of much greater significance. The progress of physics must continue; it is the 'holes' - more than a few - in the funding of physics research that must be filled.

And when, later this year, the European Space Agency launches its 'Planck' satellite, it will be a timely reminder that, in fact, every century is a century of physics. 